Pacific Journal of Mathematics

THE SINGULAR SUBMODULE OF A FINITELY GENERATED 


\title{
THE SINGULAR SUBMODULE OF A FINITELY GENERATED MODULE SPLITS OFF
}

\author{
John D. Fuelberth and Mark L. Teply
}

\begin{abstract}
A characterization is given of the finitely generated nonsingular left $R$-modules $N$ such that $\operatorname{Ext}_{R}^{1}(N, M)=0$ for every singular left $R$-module $M$. As a corollary, the rings $R$, over which the singular submodule $Z(A)$ is a direct summand of every finitely generated left $R$-module $A$, are characterized. This characterization takes on a simplified form whenever $R$ is commutative. An example is given to show that a ring $R$, over which the singular submodule $Z(A)$ is a direct summand of every left $R$-module $A$, need not be right semi-hereditary.
\end{abstract}

In this paper, all rings $R$ are assumed to be associative with an identity element, and, unless otherwise stated, all $R$-modules will be unitary left $R$-modules.

A submodule $B$ of an $R$-module $A$ is an essential submodule of $A$ if $B \cap C \neq 0$ for all nonzero submodules $C$ of $A$. A left ideal $I$ of $R$ is essential in $R$ if it is essential in $R$ as a submodule of $R$. If $A$ is an $R$-module, $Z(A)=\{a \in A \mid(0: a)$ is essential in $R\}$ is the singular submodule of $A$. $A$ is called a singular module if $Z(A)=$ $A$; and $A$ is a nonsingular module if $Z(A)=0$. A submodule $B$ of $A$ is closed in $A$ if $B$ has no proper essential extension in $A$. If $A$ is nonsingular, then a submodule $B$ of $A$ is a closed submodule of $A$ if and only if $A / B$ is a nonsingular module. A simple $R$-module $S$ is nonsingular if and only if it is projective. For an $R$-module $A$, Soc $(A)$ denotes the sum of all simple submodules of $A$ or 0 if $A$ has no simple submodules.

Motivated by a definition of Kaplansky [6], we say that an $R$-module $N$ is $U F$ if $N$ is a nonsingular module and $\operatorname{Ext}_{R}^{1}(N, M)=0$ for all singular $R$-modules $M$. An $R$-module $A$ is said to split if $Z(A)$ is a direct summand of $A$. As in [2], a ring $R$ has the finitely generated splitting property (FG.SP) if every finitely generated $R$ module splits.

We shall use the following result of Cateforis and Sandomierski [2, Proposition 1.11], which is included here for completeness.

Lemma 1. For any ring $R$, the following statements are equivalent:

(a) $R$ has FGSP.

(b) $Z(R)=0$, and every finitely generated nonsingular $R$-module is $U F$. 
An $R$-module $K$ is said to be almost finitely generated if $K=$ $U \oplus V$, where $U$ is a finitely generated $R$-module and $V=\operatorname{Soc}(V)$. Then an $R$-module $N$ is called almost finitely related if there exists an exact sequence of $R$-modules

$$
0 \longrightarrow K \longrightarrow F \longrightarrow N \longrightarrow 0,
$$

where $F$ is a finitely generated free module and $K$ is almost finitely generated.

Before stating our main results, we prove several lemmas.

Lemma 2. If $N$ is an almost finitely related $R$-module and if

$$
0 \longrightarrow K \longrightarrow F \longrightarrow N \longrightarrow 0
$$

is any exact sequence of $R$-modules with $F$ a finitely generated free module, then $K$ is almost finitely generated.

Proof. Since $N$ is almost finitely related, there exists an exact sequence of $R$-modules

$$
0 \longrightarrow K_{1} \longrightarrow F_{1} \longrightarrow N \longrightarrow 0
$$

where $F_{1}$ is a finitely generated free module and $K_{1}$ is almost finitely generated. By a result of Schanuel [9, p. 369], $K \oplus F_{1} \cong K_{1} \oplus F$. Since $K_{1}$ and $F$ are almost finitely generated, then so is $K \oplus F_{1} \cong$ $K_{1} \oplus F$. Therefore $\left(K \oplus F_{1}\right) /$ Soc $\left(K \oplus F_{1}\right)$ is finitely generated. Since

$$
\frac{K \oplus F_{1}}{\operatorname{Soc}\left(K \oplus F_{1}\right)}=\frac{K \oplus F_{1}}{\operatorname{Soc}(K) \oplus \operatorname{Soc}\left(F_{1}\right)} \cong \frac{K}{\operatorname{Soc}(K)} \oplus \frac{F_{1}}{\operatorname{Soc}\left(F_{1}\right)},
$$

then $K / \operatorname{Soc}(K)$ is also finitely generated.

Now we write $K=R x_{1}+R x_{2}+\cdots+R x_{m}+$ Soc $(K)$, where $x_{1}, x_{2}, \cdots, x_{m} \in K$. Let $W=(\operatorname{Soc}(K)) \cap\left(R x_{1}+R x_{2}+\cdots+R x_{m}\right)$. Then there exists an $R$-module $V$ such that $\operatorname{Soc}(K)=W \oplus V$. It follows that $K=\left(R x_{1}+R x_{2}+\cdots+R x_{m}\right) \oplus V$, and hence $K$ is almost finitely generated.

A finitely generated nonsingular $R$-module $N$ is called finitely generated torsion inducing (FGTI) if $N$ has the following property: If $M$ is any finitely generated $R$-module with $M / Z(M) \cong N$, then $Z(M)$ is finitely generated.

Lemma 3. Let $Z(R)=0$, and let $0 \rightarrow K \rightarrow F \rightarrow N \rightarrow 0$ be an exact sequence of $R$-modules, where $F$ is a finitely generated free module. If $N$ is nonsingular, then the following statements hold:

(a) If $N$ is FGTI and if $K / \operatorname{Soc}(K)$ is a direct sum of countably generated modules, then $N$ is almost finitely related. 
(b) If $N$ is almost finitely related, then $N$ is an FGTI module.

Proof. To show (a), we need to show that $K$ is almost finitely generated. By hypothesis, $Y=K / \operatorname{Soc}(K)=\oplus \sum_{\alpha \in} . M_{\alpha}$, where each $M_{\alpha}$ is a countably generated $R$-module. First we show that $Y$ is, in fact, countably generated also. Let $\mathscr{B}=\left\{\alpha \in \mathscr{A} \mid M_{\alpha}\right.$ contains a proper essential submodule\}. Thus if $\alpha \in \mathscr{A}-\mathscr{B}$, then $M_{\alpha}$ is a direct sum of singular simple $R$-modules or zero. For each $\alpha \in \mathscr{B}$, let $L_{\alpha}$ be a proper essential submodule of $M_{\alpha}$. Define $L=\oplus \sum_{\alpha \in} L_{\alpha}$, and let $J$ be a submodule of $K$ containing Soc $(K)$ such that $J /$ Soc $(K)=$ L. Since

$$
Z(F / J) \cong Z((F / \operatorname{Soc}(K)) /(J / \operatorname{Soc}(K))) \supseteqq Y / L \cong K / J,
$$

then $K / J$ is a singular module; but since $Z(F / K)=0$, it follows that $Z(F / J)=K / J$. By hypothesis, $N$ is a FGTI module; hence

$$
K / J \cong\left(\oplus \sum_{\alpha \in \mathscr{S}}\left(M_{\alpha} / L_{\alpha}\right)\right) \oplus\left(\oplus \sum_{\alpha \epsilon \mathscr{W}-}, M_{\alpha}\right)
$$

is a finitely generated $R$-module. Therefore all but finitely many of the $M_{\alpha}(\alpha \in \mathscr{A})$ must be 0 , and hence $K /$ Soc $(K)$ is countably generated.

Thus there exist $x_{i} \in K(i=1,2, \cdots)$ such that $K=\sum_{i=1}^{\infty} R x_{i}+$ Soc $(K)$. We will show that there exists a positive integer $m$ such that $K=\sum_{i=1}^{m} R x_{i}+\operatorname{Soc}(K)$. If this were not the case, then for each positive integer $n$, there exists a least positive integer $k(n)$ such that $x_{k: n} \notin R x_{1}+R x_{2}+\cdots+R x_{n}+$ Soc $(K)$. By Zorn's lemma, choose $K_{n}$ maximal with respect to $x_{k(n)} \notin K_{n}$ and

$$
R x_{1}+R x_{2}+\cdots+R x_{n}+\operatorname{Soc}(K) \leqq K_{n} \leqq K .
$$

It follows that $\left(R x_{k(n)}+K_{n}\right) / K_{n}$ is an essential, simple, nonprojective submodule of $K / K_{n}$. Since $K / K_{n}$ is an essential extension of a singular simple module, then $K / K_{n}$ is also a singular module.

Define $\varphi: K \rightarrow \oplus \sum_{n=1}^{\infty} K / K_{n}: x \rightarrow \sum_{n=1}^{\infty} \varphi_{n}(x)$, where $\varphi_{n}: K \rightarrow K / K_{n}$ is the natural map. If $x \in K$, then $x=\sum_{i=1}^{t} r_{i} x_{i} \in \sum_{i=1}^{t} R x_{\imath} \leqq K_{n}$ for all $n \geqq t$. Thus $\varphi_{n}(x)=0$ for all $n \geqq t$, and hence $\varphi$ is well-defined. If $H=\operatorname{ker} \varphi$, then $K / H \cong \operatorname{im} \varphi$ is not finitely generated (as $\varphi_{n}\left(x_{k(n)}\right) \neq 0$ for each integer $n$ ). Moreover, since $\operatorname{im} P$ is a submodule of the singular module $\oplus \sum_{n=1}^{\infty} K / K_{n}$, then $K / H \cong \operatorname{im} \varphi$ is also a singular module. Since $K$ is a closed submodule of $F$, then $Z(F / H)=K / H$. But then $F / H$ does not have a finitely generated singular submodule, and $(F / H) / Z(F / H) \cong F / K \cong N$. This contradicts the hypothesis that $N$ is a FGTI module. Thus $K=\sum_{\imath=1}^{m} R x_{i}+\operatorname{Soc}(K)$ for some positive integer $m$.

Now the argument used in the last paragraph of the proof of 
Lemma 2 shows that $K$ is almost finitely generated. Therefore (a) holds.

Now we prove (b). Let $M$ be a finitely generated $R$-module such that $M / Z(M) \cong N$. Let $y_{1}, y_{2}, \cdots, y_{n}$ be a set of generators of $M$, and let $F$ be a free $R$-module with basis $u_{1}, u_{2}, \cdots, u_{n}$. Then there exists a commutative diagram with exact rows

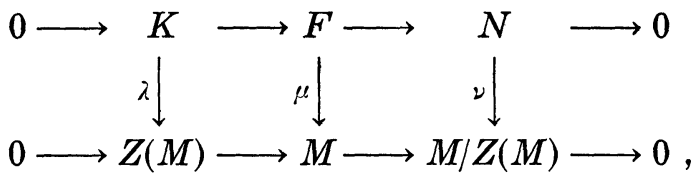

where $\mu: F \rightarrow M$ via $\mu\left(u_{i}\right)=y_{i}$ is an epimorphism and $\nu$ is an isomorphism. Then $\lambda$ must be an epimorphism. By the hypothesis and Lemma 2, $K=U \oplus V$, where $U$ is a finitely generated $R$-module and $V=\operatorname{Soc}(V)$. Since $\lambda(V)$ is isomorphic to a submodule of the nonsingular, semi-simple module $V$ and since $Z(M)$ is singular, then $\lambda(V)=0$. Thus $Z(M)$ is an epimorphic image of the finitely generated module $U$. Consequently, $Z(M)$ is a finitely generated module.

REMARKs. (1) If $R$ is a left hereditary ring, then any closed submodule $K$ of a finitely generated free module $F$ is projective. So it follows from [7, Theorem 1] that $K / \operatorname{Soc}(K)$ is a direct sum of countably generated modules. Thus for a left hereditary ring $R$, a finitely generated nonsingular $R$-module $N$ is FGTI if and only if $N$ is almost finitely related.

(2) Suppose that $N, F$, and $K$ are as in the hypothesis of Lemma 3. If $N$ is FGTI and Soc $(K)$ is essential in $K$, then $K / \operatorname{Soc}(K)$ is finitely generated. So we can conclude the following result from Lemma 3: If $R$ is a nonsingular ring with essential socle, then a finitely generated nonsingular FGTI module is almost finitely related.

(3) There seems to be some independent interest in determining when the singular submodule of a finitely generated module is finitely generated. Indeed, Pierce [8, p. 109] asks questions along this line. Lemma 3 and the first of this remark shed some light in this direction.

We shall use $h d(N)$ to denote the projective homological dimension of an $R$-module $N$.

We now need an obvious generalization of a result of Kaplansky, [6, Theorem 1]:

Lemma 4. If $N$ is a UF $R$-module, then $h d(N) \leqq 1$.

Proof. Let $N$ be a $U F R$-module, and let $M$ be any $R$-module. The exact sequence 


$$
0 \longrightarrow M \longrightarrow E(M) \longrightarrow E(M) / M \longrightarrow 0
$$

induces the exact sequence

$$
\operatorname{Ext}_{R}^{1}(N, E(M) / M) \longrightarrow \operatorname{Ext}_{R}^{2}(N, M) \longrightarrow \operatorname{Ext}_{R}^{2}(N, E(M))=0,
$$

where $E(M)$ denotes the injective hull of $M$. Since $N$ is $U F$ we have $\operatorname{Ext}_{R}^{1}(N, E(M) / M)=0$; and hence $\operatorname{Ext}_{R}^{2}(N, M)=0$ by exactness.

We now give a characterization of $U F$ modules.

Theorem 1. Let $Z(R)=0$, and let $N$ be a finitely generated $R$-module. Then $N$ is $U F$ if and only if the following conditions are satisfied:

(i) $N$ is an almost finitely related, nonsingular module.

(ii) $h d(N) \leqq 1$.

(iii) $\operatorname{Tor}_{1}^{R}\left(\operatorname{Hom}_{Z}(A, D), N\right)=0$, where $A$ is any singular $R$ module, $D$ is any divisible Abelian group, and $Z$ denotes the ring of integers.

Proof. We develop a diagram (see $\left(^{*}\right)$ ), which we use in both directions of the proof. For any finitely generated $R$-module $N$, there is an exact sequence

$$
0 \longrightarrow K \longrightarrow F \longrightarrow N \longrightarrow 0 \text {, }
$$

where $F$ is a finitely generated free $R$-module. If $D$ is any divisible Abelian group and if $A$ is any singular $R$-module, then $\operatorname{Hom}_{z}(A, D)$ is a right $R$-module. Hence there is an exact sequence

$$
\begin{aligned}
0 \longrightarrow & \operatorname{Tor}_{1}^{R}\left(\operatorname{Hom}_{Z}(A, D), N\right) \longrightarrow \operatorname{Hom}_{Z}(A, D) \otimes_{R} K \\
& \longrightarrow \operatorname{Hom}_{Z}(A, D) \otimes_{R} F .
\end{aligned}
$$

The exact sequence

$$
\operatorname{Hom}_{R}(F, A) \longrightarrow \operatorname{Hom}_{R}(K, A) \longrightarrow \operatorname{Ext}_{R}^{1}(N, A) \longrightarrow 0
$$

induces an exact sequence

$$
\begin{aligned}
0 \longrightarrow \operatorname{Hom}_{Z}\left(\operatorname{Ext}_{R}^{1}(N, A), D\right) \longrightarrow \operatorname{Hom}_{Z}\left(\operatorname{Hom}_{R}(K, A), D\right) & \longrightarrow \operatorname{Hom}_{Z}\left(\operatorname{Hom}_{R}(F, A), D\right) .
\end{aligned}
$$

It is well-known $[1$, p. 120] that there exists a homomorphism $\psi$ and an isomorphism $\beta$ making the following diagram commutative:

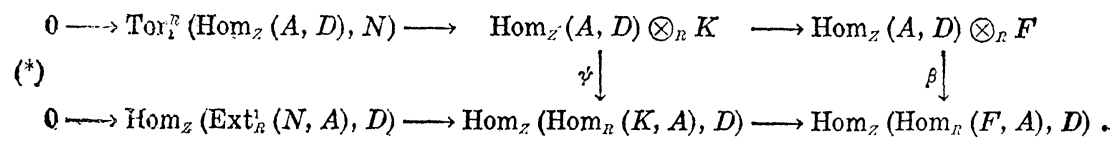


"only if": Let $N$ be a finitely generated $U F R$-module. Then there exists an exact sequence

$$
0 \longrightarrow K \longrightarrow F \longrightarrow N \longrightarrow 0
$$

of left $R$-modules, where $F$ is a finitely generated free module. By Lemma $4, K$ is a projective $R$-module; thus $K=\oplus \sum_{\alpha \in \ldots} K_{\alpha}$, where each $K_{r}$ is countably generated by [7, Theorem 1]. Since

$$
\frac{K}{\operatorname{Soc}(K)}=\frac{\bigoplus \sum_{\alpha \epsilon} K_{\alpha}}{\bigoplus \sum_{\alpha \varepsilon \mathscr{A}} \operatorname{Soc}\left(K_{\alpha}\right)} \cong \oplus \sum_{\alpha \varepsilon}, \frac{K_{\alpha}}{\operatorname{Soc}\left(K_{\alpha}\right)},
$$

then $K /$ Soc $(K)$ is a direct sum of countably generated $R$-modules. Since a $U F$ module is FGTI, then Lemma 3 (a) implies that $N$ is almost finitely related, i.e., (i) holds.

Lemma 4 implies that $h d(N) \leqq 1$; so (ii) holds.

Now we show that (iii) holds. Let $A, D, F$, and $K$ be chosen as in (*). Then by (i), $K=U \oplus V$, where $U$ is finitely generated and $V=\operatorname{Soc}(V)$. But for any nonsingular simple $R$-module $S, \operatorname{Hom}_{R}(S, A)=$ 0 (as $A$ is singular). Thus by [1, VI. Prop. 5.2], $\operatorname{Hom}_{Z}(A, D) \otimes_{I} S \cong$ $\operatorname{Hom}_{\%}\left(\operatorname{Hom}_{R}(S, A), D\right)=0$. Therefore $\operatorname{Hom}_{z}(A, D) \otimes_{R} V=0$, and $\mathrm{Hom}_{R}(V, A)=0$. Hence there exist obvious isomorphisms $\sigma$ and $\tau$ making the diagram

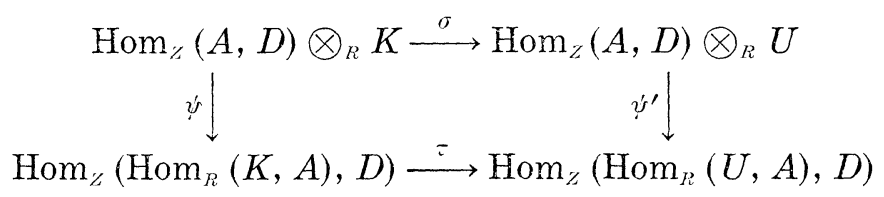

commute, where $\psi^{\prime}$ is the restriction of is in $\left(^{*}\right)$ to $\operatorname{Hom}_{\%}(A, D) \otimes_{R} U$. By [1, VI. Prop. 5.2] $\gamma^{\prime} r^{\prime}$ is an isomorphism; whence $\gamma^{\prime} s$ is forced to be an isomorphism also. By the commutativity of $(*)$ and the fact that $\operatorname{Ext}_{R}^{\prime}(N, A)=0$, it is now easy to obtain $\operatorname{Tor}_{1}^{R}\left(\operatorname{Hom}_{\%}(A, D), N\right)=0$.

"if": Let $A, D, F, K$ be as in ("). Since $h d(N) \leqq 1$ and $N$ is almost finitely related, then $K$ is an almost finitely generated projective $R$-module. By the argument used in the preceding paragraph, $\gamma$ is an isomorphism in $(*)$. From the commutativity of $\left(^{*}\right)$ and the fact that $\operatorname{Tor}_{1}^{\prime \prime}\left(\operatorname{Hom}_{z}(A, D), N\right)=0$, we now obtain $\operatorname{Hom}_{\%}\left(\operatorname{Ext}_{z}^{\prime}(N, A), D\right)=$ 0 . Since $D$ is any divisible Abelian group, then $\operatorname{Ext}_{l}^{1}(N, A)=0$ for every singular module $A$. Thus $N$ is a $U F$ module.

As a corollary, we have the following result for left hereditary rings:

Corollary 1. Let $R$ be a left hereditary ring whose maximal 
quotient ring ${ }_{R} Q$ (see [3], [11]) is R-flat. Then the following statements are equivalent for any finitely generated nonsingular $R$ module $N$ :

(a) $N$ is a UF module.

(b) $N$ is almost finitely related.

(c) $N$ is a FGTI module.

Proof. The equivalence of (b) and (c) is clear from Remark (1) following Lemma 3. The equivalence of (a) and (b) will follow immediately from Theorem 1 if we show that the ring hypothesis implies every nonsingular $R$-module is $R$-flat. But this follows from [11, Cor. 2.5] and [11, Theorem 2.1].

An immediate consequence of Lemma 1 and Theorem 1 is the following characterization of FGSP:

Co ROLLARY 2. A ring $R$ has FGSP if and only if the following statements hold:

(a) $Z(R)=0$.

(b) Every finitely generated nonsingular $R$-module is almost finitely related.

(c) $h d(N) \leqq 1$ for every finitely generated nonsingular $R$-module $N$.

(d) $\operatorname{Tor}_{1}^{R}\left(\operatorname{Hom}_{Z}(A, D), N\right)=0$, where $N$ is any finitely generated nonsingular $R$-module, $D$ is any divisible Abelian group, and $Z$ denotes the ring of integers.

Combining Corollaries 1 and 2, the reader can easily see that a left hereditary ring $R$, whose maximal left quotient $\operatorname{ring}{ }_{R} Q$ is flat, has FGSP if and only if every finitely generated nonsingular $R$-module is almost finitely related. We shall see in Corollary 6 that Corollary 2 also takes on a particularly nice form whenever $R$ is a commutative ring.

A submodule $K$ of an $R$-module $M$ is said to be an almost summand of $M$ if $K=U \oplus V$, where $U$ is a direct summand of $M$ and $V=\operatorname{Soc}(V)$. The next theorem gives a relationship between $U F$ $R$-modules and almost summands of free $R$-modules.

THEOREM 2. Let $Z(R)=0$, and let $N \cong F / K$ be a finitely generated nonsingular $R$-module, where $F$ is a finitely generated free $R$ module. If $K$ is an almost summand of $F$, then $N$ is UF. Moreover, if $N$ is R-flat, then the converse holds.

Proof. To prove the first statement, it suffices to show that any homomorphism $f: K \rightarrow A$ can be lifted to a homomorphism $g: F \rightarrow A$, where $A$ is any singular module. Now $K=U \oplus V$, where 
$F=U \oplus W$ for some submodule $W$ of $F$ and $V=$ Soc $(V)$. Since $Z(A)=A$ and $Z(K)=0$, then $f(\operatorname{Soc}(K))=0$. If $x \in K \cap W$, it follows from the direct sum decompositions that $x \in \operatorname{Soc}(K)$, and hence $f(x)=$ 0 . So the desired lifting of $f$ is given by $g(u+w)=f(u)$ for all $u \in U$ and all $w \in W$.

Now assume $N$ is an $R$-flat $U F$ module. By Theorem $1, K=$ $U \oplus V$, where $U$ is finitely generated and $V=\operatorname{Soc}(V)$ is projective. Then there is an exact sequence

$$
0 \longrightarrow K / U \longrightarrow F / U \longrightarrow F / K \longrightarrow 0
$$

with $K / U$ and $F / K R$-flat. Thus $F / U$ is also $R$-flat. But $F / U$ is finitely related (see [5, p. 459]) and therefore projective by [5, p. 459]. Consequently $U$ is a direct summand of $F$, and $K=U \oplus V$ is an almost summand of $F$.

The following corollary is an immediate consequence of Lemma 1 and Theorem 2.

CoROllary 3. If $Z(R)=0$ and if every closed submodule of a finitely generated free $R$-module $F$ is an almost summand of $F$, then $R$ has FGSP. Moreover, if every (finitely generated) nonsingular $R$-module is flat, then the converse holds.

The next corollary is a partial generalization of [11, Corollary 2.7].

COROLlaRY 4. If $R$ is a right semi-hereditary ring having a maximal left quotient ring $Q$ (see [3], [11]), which is a two-sided quotient ring of $R$, then the following statements are equivalent:

(a) $R$ has FGSP.

(b) $Z(R)=0$, and every closed submodule of a finitely generated free $R$-module $F$ is an almost summand of $F$.

Proof. By Corollary 3, we need to show that if $R$ has FGSP, then every nonsingular $R$-module is flat. Since $Z(R)=0$ by Lemma 1 and since $Q$ is two-sided, then every finitely generated nonsingular $R$-module is torsionless by [3, Theorem 1.1]. However $R$ is right semi-hereditary; hence every torsionless $R$-module is flat by [5, Theorem 4.1].

COROLlaRY 5. Let $R$ be a commutative ring with $Z(R)=0$. Let $N \cong F / K$, where $F$ is a finitely generated free $R$-module. Then $N$ is UF if and only if $N$ is a nonsingular module and $K$ is an almost summand of $F$. 
Proof. By Theorem 2, it suffices to show that any $U F R$ module is $R$-flat. But this follows from the proof of the corollary to [2, Proposition 1.11].

Pierce [8, p. 109] asks when a finitely generated module over a commutative regular ring splits. Corollary 5 sheds some light in this direction. Moreover, since the hypothesis, " $R$ is a commutative ring with $Z(R)=0$," is used only to establish that nonsingular modules are flat, the conclusion of Corollary 5 holds true for any regular ring $R$. Corollary 5 also generalizes [10, Theorem 3.3], which deals with the structure of rings for which cyclic modules split.

In [2] Cateforis and Sandomierski have suggested the question of determining all commutative rings with FGSP. The final corollary extends [10, Theorem 3.3] to give an answer to this question.

CoRollary 6. If $R$ is a commutative ring, then the following statements are equivalent:

(a) $R$ has FGSP.

(b) $Z(R)=0$, and every closed submodule of a finitely generated free $R$-module $F$ is an almost summand of $F$.

(c) $R$ is semi-hereditary, and every finitely generated nonsingular module is almost finitely related.

Proof. The equivalence of (a) and (b) follows from Lemma 1 and Corollary 5. In view of the corollary to [2, Proposition 1.11], (c) is an immediate consequence of (a) and (b). Assuming (c), the last two sequences in the proof of Corollary 4 show that all nonsingular modules are flat. Hence (b) follows by a slight modification of the argument used in the last part of the proof of Theorem 2.

The authors conjecture that a ring $R$ has FGSP if and only if $Z(R)=0$ and every closed submodule of a finitely generated free module $F$ is an almost summand of $F$.

In view of the preceding corollaries and the corollary to [2, Proposition 1.11], the reader might conjecture that the messy "Tor condition" in Corollary 2 (d) can be replaced by the nicer condition, " $R$ is right semi-hereditary," or by the stronger condition, "all nonsingular $R$-modules are flat." However, the following example shows that a ring $R$ with FGSP need not be right semi-hereditary.

ExAmple. Let $F$ be a field, and let $T$ be the $F$-subalgebra of $\prod_{n=1}^{\infty} F^{(n)}$ generated by $\oplus \sum_{n=1}^{\infty} F^{(n)}$ and the identity of $\prod_{n=1}^{\infty} F^{(n)}$, where $F^{(n)} \cong F$ for all $n$. Let $I=\oplus \sum_{n=1}^{\infty} F^{(n)}$, and let $S=T / I$. If $R$ is the ring of all $2 \times 2$ matrices of the form 


$$
\left\{\left(\begin{array}{ll}
a & b \\
0 & c
\end{array}\right) \mid a, b \in S ; c \in T\right\},
$$

then Chase [4, Proposition 3.1] has shown that $R$ is a left semihereditary ring, which is not a right semi-hereditary ring. Hence $Z(R)=0$, and it is straight forward to check that the only proper essential left ideal of $R$ is the maximal left ideal

$$
J=\left\{\left(\begin{array}{ll}
a & b \\
0 & c
\end{array}\right) \mid a, b \in S ; c \in I\right\} .
$$

Thus if $A$ is any singular $R$-module, then $A$ is a direct sum of copies of the simple module $R / J$. It follows that each singular module is injective, and hence every $R$-module splits. Thus $R$ has FGSP, but $R$ is not right semi-hereditary.

Added in proof. K. R. Goodearl has constructed an example (unpublished) which shows that the conjecture following Corollary 6 is not true.

\section{REFERENCES}

1. H. Cartan, and S. Eilenberg, Homological Algebra, Princeton, N. J., Princeton University Press, 1956.

2. V. C. Cateforis, and F. L. Sandomierski, The singular submodule splits off, J. of Algebra 10 (1968), 149-165.

3. - On modules of singular submodule zero, Canad. J. Math., 23 (1971), $345-354$.

4. S. U. Chase, A generalization of the ring of triangular matrices, Nagoya Math. J., 18 (1961), 13-25.

5. — Direct product of modules, Trans. Amer. Math. Soc., 97 (1960), 457-473.

6. I. Kaplansky, The splitting of modules over integral domains, Archiv. der Math., 13 (1962), 341-343.

7. —_ Projective modules, Annals of Math. 68 (1958), 372-377.

8. R. S. Pierce, Modules over commutative regular rings, Memoirs Amer. Math. Soc., 70, 1967.

9. R. G. Swan, Groups with periodic cohomology, Bull. Amer. Math. Soc. 65 (1959), 368-370.

10. M. L. Teply, The torsion submodule splits off II, (to appear).

11. D. R. Turnidge, Torsion theories and semi-hereditary rings, Proc. Amer. Math. Soc., 24 (1970), 137-143.

Received December 9, 1970 and in revised form March 22, 1971. This work was partially supported by a University of Florida Graduate School Post Doctoral Fellowship.

UNIVERSITY OF FLORIDA 


\section{PACIFIC JOURNAL OF MATHEMATICS}

\section{EDITORS}

H. SAMELSON

Stanford University

Stanford, California 94305

C. R. HOBBY

University of Washington

Seattle, Washington 98105
J. DugundJI

Department of Mathematics

University of Southern California

Los Angeles, California 90007

RICHARD ARENS

University of California

Los Angeles, California 90024

\section{ASSOCIATE EDITORS}

E. F. BeCKENBACH

B. H. NeumanN

F. WOLF

K. YosHIDA

\section{SUPPORTING INSTITUTIONS}

UNIVERSITY OF BRITISH COLUMBIA

CALIFORNIA INSTITUTE OF TECHNOLOGY

UNIVERSITY OF CALIFORNIA

MONTANA STATE UNIVERSITY

UNIVERSITY OF NEVADA

NEW MEXICO STATE UNIVERSITY

OREGON STATE UNIVERSITY

UNIVERSITY OF OREGON

OSARA UNIVERSITY
UNIVERSITY OF SOUTHERN CALIFORNIA STANFORD UNIVERSITY

UNIVERSITY OF TOKYO

UNIVERSITY OF UTAH

WASHINGTON STATE UNIVERSITY

UNIVERSITY OF WASHINGTON

AMERICAN MATHEMATICAL SOCIETY

NAVAL WEAPONS CENTER

Printed in Japan by International Academic Printing Co., Ltd., Tokyo, Japan 


\section{Pacific Journal of Mathematics}

\section{Vol. 40, No. $1 \quad$ September, 1972}

Alex Bacopoulos and Athanassios G. Kartsatos, On polynomials

approximating the solutions of nonlinear differential equations........

Monte Boisen and Max Dean Larsen, Prüfer and valuation rings with zero

divisors ..........................................

James J. Bowe, Neat homomorphisms

David W. Boyd and Hershy Kisilevsky, The Diophantine equation

$$
u(u+1)(u+2)(u+3)=v(v+1)(v+2) \ldots \ldots \ldots \ldots \ldots \ldots \ldots
$$

George Ulrich Brauer, Summability and Fourier analysis ...............

Robin B. S. Brooks, On removing coincidences of two maps when only one,

rather than both, of them may be deformed by a homotopy ............

Frank Castagna and Geert Caleb Ernst Prins, Every generalized Petersen

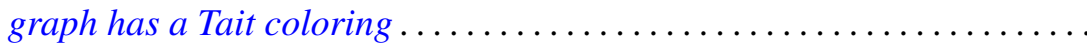

Micheal Neal Dyer, Rational homology and Whitehead products ..........

John Fuelberth and Mark Lawrence Teply, The singular submodule of a

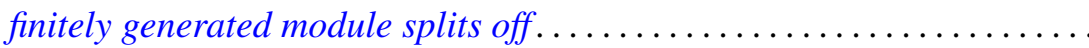

Robert Gold, $\Gamma$-extensions of imaginary quadratic fields ............ 83

Myron Goldberg and John W. Moon, Cycles in k-strong tournaments.......

Darald Joe Hartfiel and J. W. Spellmann, Diagonal similarity of irreducible

matrices to row stochastic matrices...............

Wayland M. Hubbart, Some results on blocks over local fields ..

Alan Loeb Kostinsky, Projective lattices and bounded homomorphisms....

Kenneth O. Leland, Maximum modulus theorems for algebras of operator

valued functions ...

Jerome Irving Malitz and William Nelson Reinhardt, Maximal models in the

language with quantifier "there exist uncountably many" ..

John Douglas Moore, Isometric immersions of space forms in space

forms.

Ronald C. Mullin and Ralph Gordon Stanton, A map-theoretic approach to

Davenport-Schinzel sequences ....................

Chull Park, On Fredholm transformations in Yeh-Wiener space. .

Stanley Poreda, Complex Chebyshev alterations ..............

Ray C. Shiflett, Extreme Markov operators and the orbits of Ryff. ...

Robert L. Snider, Lattices of radicals .....................

Ralph Richard Summerhill, Unknotting cones in the topological

category ................................

Charles Irvin Vinsonhaler, A note on two generalizations of $\mathrm{QF}-3 \ldots \ldots 229$

William Patterson Wardlaw, Defining relations for certain integrally

parameterized Chevalley groups...................

William Jennings Wickless, Abelian groups which admit only nilpotent

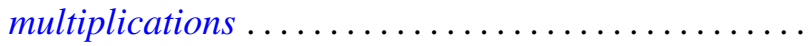

